\title{
Multi-photon Absorption in Amyloid Protein Fibres
}

\author{
Piotr Hanczyc ${ }^{\mathrm{a}, \mathrm{b}}$, Marek Samoc ${ }^{\mathrm{b}}$, Bengt Norden ${ }^{\mathrm{a} *}$ \\ ${ }^{a}$ Department of Chemical and Biological Engineering, Chalmers University of Technology, \\ SE-41296 Gothenburg, Sweden \\ ${ }^{b}$ Institute of Physical and Theoretical Chemistry, Wroclaw University of Technology, 50-370, \\ Wroclaw, Poland \\ *corresponding author: norden@chalmers.se
}

Fibrilization of peptides leads to formation of amyloid fibres that in large aggregates are responsible for such diseases as Alzheimer's and Parkinson's ${ }^{1-4}$. We show here that amyloids possess strong non-linear optical absorption which is not present in native nonfibrillized protein. Z-scan ${ }^{5}$ and pump-probe experiments indicate that insulin and lysozyme $\beta$-amyloids, as well as $\alpha$-synuclein fibres, exhibit either two-photon, threephoton or higher multi-photon absorption processes, depending on the wavelength of light. We propose that the enhanced multiphoton absorption is due to a cooperative mechanism $^{6}$ involving through-space dipolar coupling between excited states of aromatic amino acids densely packed in the fibrous structures. The finding is creating the opportunity for non-linear optical techniques to detect and study amyloid structures and is also suggesting that new protein-based materials with sizable multiphoton absorption could be designed for specific applications in nanotechnology, photonics and optoelectronics. 
Fibrilization of peptides or polypeptides leads to formation of amyloid structures ${ }^{1}$. The process is promoted by certain mutations that affect protein folding which may result in erratic structures, such as self-assembled isolable aggregates believed responsible for various diseases including the Alzheimer's, the Parkinson's and the (contagious) Creutzfeldt-Jakob disease $^{2-4,7}$. Amyloid fibrils are generally composed of antiparallel $\beta$-sheets oriented perpendicular to the long-axis of the fibril which can be as long as several micrometres but with a diameter of only some $8-10 \mathrm{~nm}^{8}$. For certain systems mixed unstructured $\alpha$-helixes and $\beta$-sheet fibrous structures may co-exist in equilibrium (e.g. for $\alpha$-synuclein). Such natural biopolymer aggregates often exhibit unusual material properties: pull strength comparable to steel, mechanical shear stiffness similar to that of silk and extreme persistence length and mechanical rigidity ${ }^{9}$. Amyloid fibrils have attracted considerable interest lately because of their proven medicinal relevance, both as for underlying mechanistic relations to disease and for the development of new diagnostic tools. From another angle, protein fibrils may also be considered a new class of self-assembling nanoscale biopolymer materials with properties that could be useful, for example, in various photonics applications as suggested by the non-linear phenomena reported here.

We report the discovery that amyloid fibrils exhibit enhanced multiphoton absorption properties apparently directly related to the fibrilization. The parameters characterizing the non-linear absorption and its character (Figure 1) were determined using the Z-scan technique $^{5}$ in the wavelength range from 530 to $950 \mathrm{~nm}$, and the fibrils were compared with native protein monomers of equimolar concentrations. Three kinds of fibre-forming proteins were investigated and all, to greater or less extent, were found to exhibit enhanced non-linear properties. Insulin fibrils were also examined by the pump-probe technique at $800 \mathrm{~nm}$ and confirmed to display signals that can be attributed to enhanced multiphoton absorption (Figure S6). 


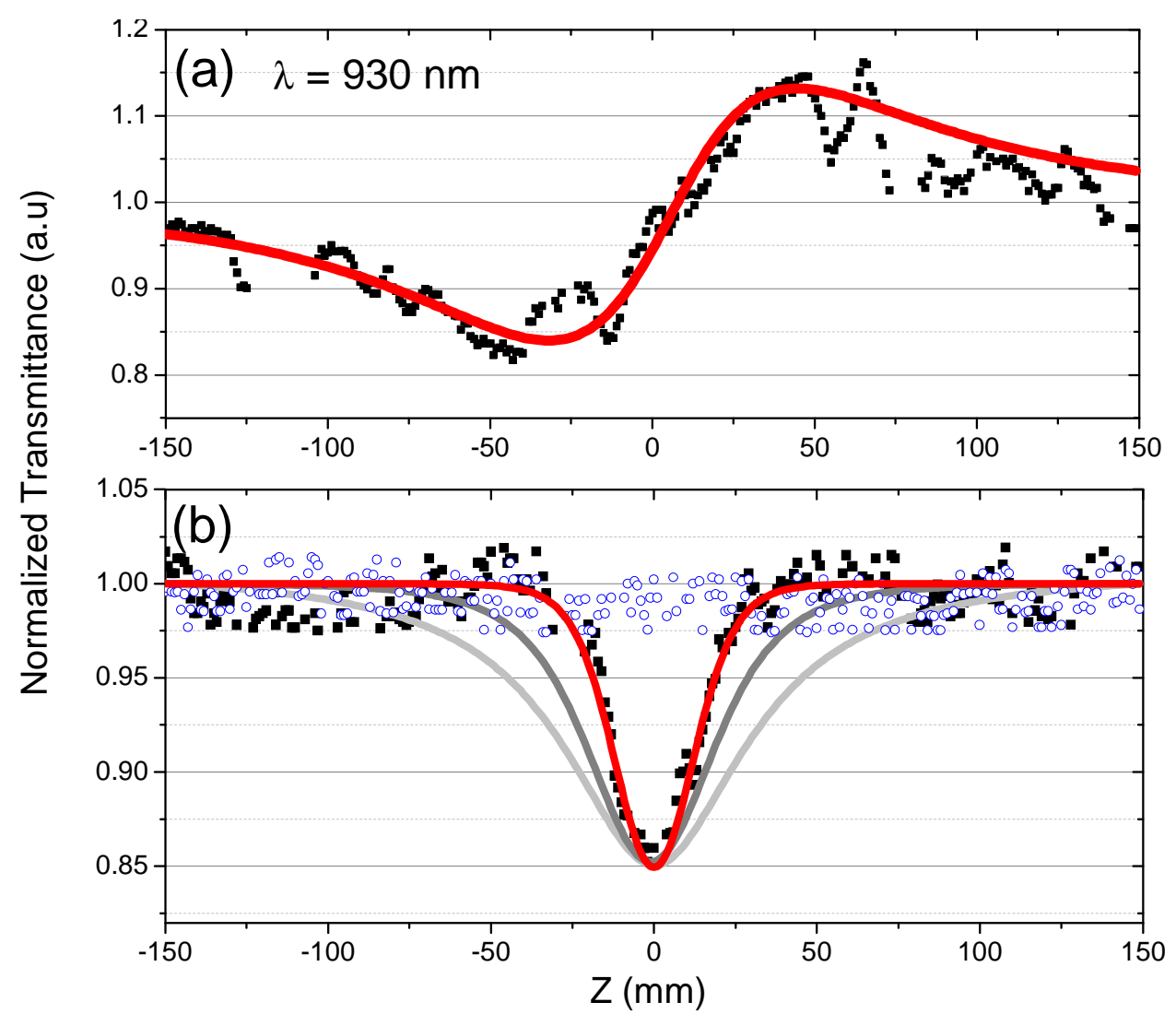

Fig 1. Example of Z-scan measurement of non-linear refractive index (a) and non-linear absorption coefficient (b) of amyloid sample. Femtosecond Z-scan using $100 \mathrm{GW} / \mathrm{cm}^{2}$ irradiation at $930 \mathrm{~nm}$ with closed (a) and open (b) apertures. Amyloid fibrils (squares) are compared to native protein (circles). Theoretical fits are shown for two-photon absorption (2PA, light-grey solid curve), three-photon absorption (3PA, dark-grey solid curve) and five photon absorption (5PA, red solid curve). 


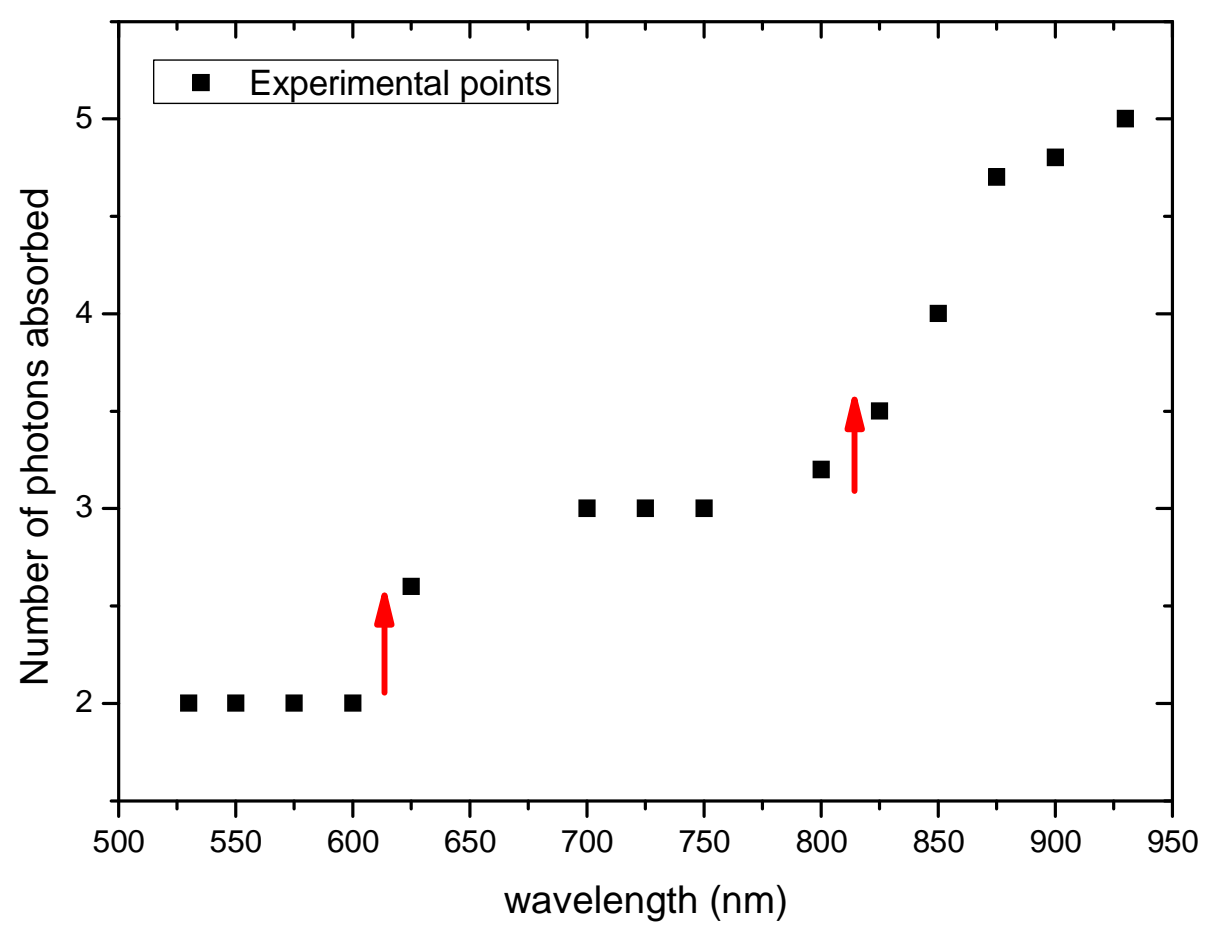

Fig 2. Wavelength dependence of exponent $n$ (the apparent number of photons taking part in the non-linear absorption process) defined from $\mathrm{d} I / \mathrm{d} z=-k I^{n}$ with $I$ denoting light intensity and $z$ propagation distance. Note the steps marked with vertical arrows: below $625 \mathrm{~nm}$, two-photon absorption and below $850 \mathrm{~nm}$, three-photon absorption.

Pure two-photon absorption (2PA) was only observed at the shortest wavelengths applied in our experiments, as judged by the criterion of the fit between experimental open aperture scan and theoretical line shapes for different numbers of photons assumed to be absorbed simultaneously (Figure 1 and S2 - S5 in Supplementary Information) ${ }^{10}$. These results are summarized in Figure 2. It can be seen that 2PA is dominant in the range 525-600 nm, while a three-photon absorption (3PA) process appears to dominate in the range 700-750 $\mathrm{nm}$ in agreement, for example, with the position of the lowest single photon transition of tyrosine occurring at $275 \mathrm{~nm}$. At the longest wavelengths the value of $n$ needed to fit the experimental data is close to 5 . 
Figure 3 shows non-linear absorption spectra presented as two-photon and three-photon absorption cross-sections versus wavelength, respectively, where appropriate, and as an apparent two-photon cross section (see SI for definition) corresponding to the typical light intensity of measurements $\left(100 \mathrm{GW} / \mathrm{cm}^{2}\right)$ for regions where neither clean 2PA nor 3PA behaviour was observed. Within the region where a two-photon process dominates there is a prominent maximum at $550 \mathrm{~nm}$ with $\sigma_{2}=2600 \mathrm{GM}$ (calculated per insulin monomer, $1 \mathrm{GM}=$ $10^{-50} \mathrm{~cm}^{4} \mathrm{~s}$ ), in good agreement with the true linear (one-photon absorption) spectrum replotted in the figure versus the wavelength number multiplied by a factor of two.

Table 1

\begin{tabular}{|l|l|l|l|l|l|}
\hline $\begin{array}{l}\text { Amyloid fibril } \\
\text { type }\end{array}$ & $\begin{array}{l}\text { Number of aromatic } \\
\text { amino acids in monomer } \\
\text { unit (Y, F, W) }\end{array}$ & $\begin{array}{l}\text { Molecular weight } \\
\text { of monomer unit } \\
{[\mathrm{g} / \mathrm{mol}]}\end{array}$ & $\begin{array}{l}\text { 2PA max. } \\
\text { wavelength }\end{array}$ & $\sigma_{2}[\mathrm{GM}]$ & $\sigma_{2} / \mathrm{M}$ \\
\hline insulin & $4 \mathrm{x} \mathrm{Y,3x} \mathrm{F,0x} \mathrm{W}$ & 5733,5 & $550 \mathrm{~nm}$ & 2600 & 0.45 \\
\hline lysozyme & $3 \times \mathrm{Y}, 3 \times \mathrm{F}, 6 \mathrm{x}$ W & 14307 & $570 \mathrm{~nm}$ & 1640 & 0.11 \\
\hline$\alpha$-synuclein & $4 \mathrm{x} \mathrm{Y,2x} \mathrm{F,0x} \mathrm{W}$ & 14460 & $550 \mathrm{~nm}$ & 5250 & 0.36 \\
& & & & & \\
\hline
\end{tabular}

To appreciate the strength of the non-linear optical response, and compare it among various species, it is useful to consider certain merit factors, e.g., to scale the two-photon absorption cross-section by the molecular weight ${ }^{11-13}$ of absorbing species. In the case of insulin fibrils we find that at $550 \mathrm{~nm} \sigma_{2} / \mathrm{M}=0.45$, a value three orders higher than for pure tyrosine $\sigma_{2} / \mathrm{M}=0.13 \times 10^{-3},{ }^{14}$ and comparable to those reported for good two-photon dyes. This is indeed an unexpectedly large value, especially recalling that no two-photon absorption could 
be recorded under the same conditions in the case of native insulin protein i.e. the same amino acid sequence.

In order to get further support for the hypothesis that it is the fibrillar state that is specifically enhancing multiphoton absorption, we performed the same experiments also on lysozyme and $\alpha$-synuclein fibrils which have different compositions of aromatic residues (see Table 1). Both showed enhanced 2PA but, upon scaling, lysozyme was found to be a 3-4 times weaker twophoton absorber than the richer in tyrosine proteins, $\alpha$-synuclein and insulin.

In the wavelength range of the dominant three-photon process (from 700 to $750 \mathrm{~nm}$ ) a maximum at $725 \mathrm{~nm}$ for insulin (Figure S3) and $750 \mathrm{~nm}$ for $\alpha$-synuclein (Figure S4) corresponds to the three-photon absorption cross sections $\sigma_{3}=3.3 \times 10^{-77} \mathrm{~cm}^{6} \mathrm{~s}^{2}$ and $\sigma_{3}=1.4 \mathrm{x}$ $10^{-76} \mathrm{~cm}^{6} \mathrm{~s}^{2}$, respectively, which are, again, notably high values. Some caution is in order when assigning the origin of the effect, i.e. the three-photon active transition, as the data points corresponding to the 3PA spectrum do not perfectly follow the pattern of the one-photon absorption spectrum wavelength-scaled by a factor of three: a substantial blue shift is seen when comparing the spectra. Note that a blue-shift is predicted for side-by-side exciton models for two-photon absorption (see Supplementary Information). The data collected in the intermediate region and at longer wavelengths $2<\mathrm{n}<3$ and $3<\mathrm{n}<5$ (Figure 2) where a mixture of two or more processes may occur also show appreciable multi-photon absorption. It is interesting to realize that the multiphoton absorption processes actually lead to the fraction of incident photons absorbed comparable to that expected for strong two-photon absorbers at the same light intensities.

Our results for amyloids stand out when one notes that among natural biopolymers only rather modest non-linear optical properties have been reported so far, for $\mathrm{DNA}^{15}$ and isolated aromatic amino acids ${ }^{16}$. It is, therefore, not surprising that proteins in their native states show negligible $2 \mathrm{PA}^{14}$. Probably as a result of a relatively small number of aromatic amino acids in 
protein sequence and, hence generally, quite large inter-molecular separations between them in the monomer protein structure there is no particular communication between the aromatic amino acids in the non-fibrillized state. However, influence of protein conformation on 2PA has been considered previously as an important factor ${ }^{14,16}$. The non-linear absorption exhibited by the aggregated state of proteins is reminiscent of non-linearity enhancements reported for extended $\pi$-electron systems like in dendrimers ${ }^{17-19}$ and porphyrins ${ }^{20}$ where strong enhancement of $2 \mathrm{PA}$ is observed upon progressing from the zeroth to the first generation of dendrimer or conjugation of porphyrins into ladder type structures. Unfortunately, the complexity of the amyloid fibril systems, as compared to the structurally relatively well-defined dendrimers or porphyrins, together with a general lack of structural information about their internal organization of chromophores in the fibres, makes it difficult to deduce the mechanisms and parameters of the proteins that will determine the overall nonlinear response. However, in contrast to the dendrimers and porphyrins, our protein fibrils are all lacking $\pi$-conjugation. This fact together with the generally very large separation of the aromatic chromophores when counted along the peptide chains, make us dismiss the throughbond cooperative effects as major origin of enhanced 2PA. As inferred from a more detailed discussion in Supplementary Information, instead intermolecular interactions are operative and it is proposed that an exciton type of interaction is the origin of strong two-photon absorption.

Aggregation-induced enhancements of 2PA were recently reviewed and discussed in terms of co-operative effects of intra- and inter-molecular charge transfer transitions ${ }^{6}$. It is plausible that similar cooperative mechanisms may be also responsible for the strong enhancement of non-linear optical response in compacted structures of amyloid fibrils leading to large nonlinear absorption cross section values. Our results indicate that also the sequence composition is important, and probably possible modifications of amino acids such as nitration or 
phosphorylation may influence cooperative enhancement. We note that lysozome that contains more trypthophans dominating one-photon absorption (OPA) spectrum in a sequence is found to exhibit significantly weaker $2 \mathrm{PA}$ than insulin and $\alpha$-synuclein. However, this is not surprising since trypthophan itself has only weak $2 \mathrm{PA}$ when compared to tyrosine ${ }^{16}$ present in larger amounts there. Thus, the scaled 2PA property indicates that tryptophan-rich amyloids do not a show non-linear absorption enhanced as effectively as tyrosine-rich fibril structures. Another aspect to note may be distance variations between tyrosines in a monomer sequence, although we consider that of minor importance for a cooperative mechanism that is based on through-space rather than through-bond interaction. Comparing tyrosine-rich amyloids it is reasonable that 2PA of insulin is larger than that of $\alpha$-synuclein because the tyrosine content is higher and the monomer unit is almost three times smaller allowing denser packing. To sum up, the three different proteins in fibril state, insulin, lysozome and $\alpha$ synuclein, all exhibit multiphoton properties that appear to be related to tyrosine content, whereas other aromatic amino acids seem to play less important roles in inducing enhanced non-linear response. Finally, linear dichroism studies of tyrosine-containing amyloid fibres indicate a common behaviour: a preferential orientation of the transition moment of the first symmetry-forbidden 1PA transition $\mathrm{L}_{\mathrm{b}}$ along the fibre axis and more perpendicular orientation of the next transition dipole moment $\mathrm{L}_{\mathrm{a}}$. By contrast, the $\mathrm{L}_{\mathrm{b}}$ transition, occurring at $275 \mathrm{~nm}$, is allowed in 2PA and the cause of reasonably strong non-linear effects in isolated tyrosines.

In the case of amyloid fibrils no significant conjugation or delocalization of electrons occurs along the peptide chain and any further enhancement effects, we propose, therefore must be due instead to intermolecular (through-space) interactions. Based on more detailed arguments given in Supplementary Information we suggest that the cooperative effects in the amyloid fibres are predominantly of excitonic nature such as the symmetric combinations of $\mathrm{L}_{b}$ transition dipole moments schematically illustrated in scheme 1. 


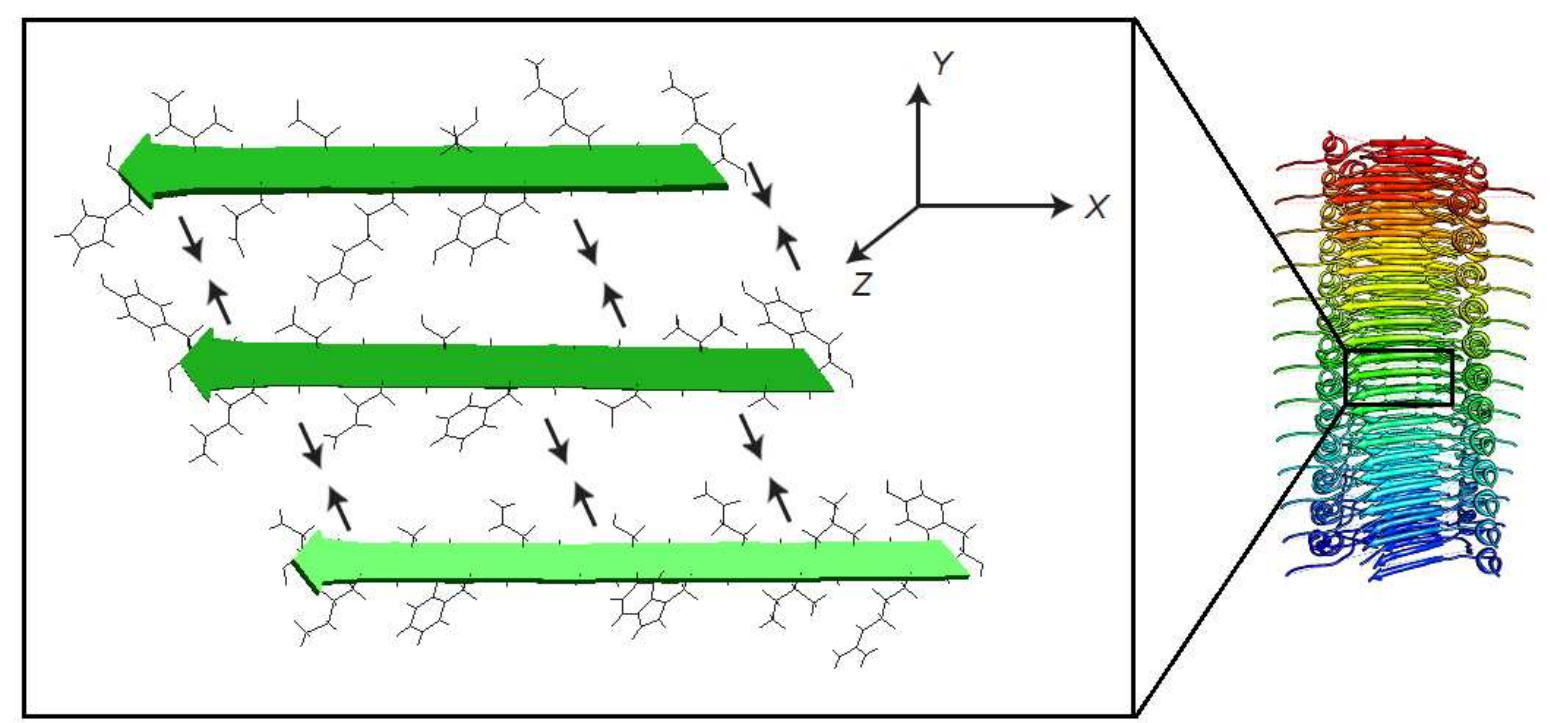

Scheme 1. Schematic illustration of proposed cooperative enhancement of multiphoton absorption by exciton coupling among tyrosins that may interact in all three dimensions in the amyloid structure.

Existing preparation routes for amyloids ${ }^{21}$, where length, thickness and amino acid composition can be reasonably accurately predicted and controlled at an early stage of assembly, are appealing for the fabrication of non-linear absorbers with tunable properties. The material properties of such biopolymers could make them excellent candidates for developing new non-linear self-assembling nano-templates for, e.g., plasmonic nanoparticle wires $^{22}$ or, if combined with addressable DNA origami grids ${ }^{23}$, functional opto-electronic components in molecular "integrated circuits".

In parallel with advancing the fundamental insight into structure and physical properties of amyloid fibrils, these often also represent a state of misfolding and subsequent aggregation that can occur among virtually any protein in nature ${ }^{24}$. There is a demand for localizing positions along the amino acid sequence where proteins tend to aggregate which could prime the development of serious diseases. There are a few sensitive methods for detecting the specific aggregation that lie behind formation of toxic fibres and the kinetics of their 
formation. But most of them are invasive and require additional sample treatment such as labeling with organic dyes ${ }^{25}$ or metals ${ }^{26}$ that is often cytotoxic and not applicable in vivo. An example of a non-invasive absorption-based method for fibril formation detection is linear electronic circular dichroism $(\mathrm{CD})^{27}$ and we envisage that our discovery may open an access to an alternative multiphoton-based methods such as "non-linear circular dichroism" $(\mathrm{NLCD})^{28}$. NLCD could open the possibility to investigate conformational changes of proteins just like linear electronic $\mathrm{CD}$ (for example, misfolding) but in the near infrared transparent region above $700 \mathrm{~nm}$, where cells and tissues are not prone to photo-damage and exhibit less light scattering. The cooperativity enhancing non-linear absorption of fibres may be also useful in photo-acoustic tomography ${ }^{29}$ where in multiphoton process only aggregation states would be visualized whereas non-aggregated globular proteins would remain optically invisible.

Envisaging using advanced multiphoton technologies both for diagnostic as well as therapeutic, even prophylactic, methodologies sounds exciting but also brings new kinds of limitations and problems. Other fibrillar proteins such as collagen known to exhibit second harmonic generation ${ }^{30}$ may interfere with detection of amyloids. Thus, further research concerning sensitivity and background effects is necessary. 




Figure 3 Diagram representing the values of non-linear absorption at different wavelength in visible and infrared region. Two-photon absorption of amyloid fibrils (black squares) between 530 and 600 $\mathrm{nm}$, together with one-photon spectrum (solid grey) re-plotted from normal absorption spectrum with the wavelength multiplied by a factor of two and normalized to the same peak height for comparison. Three-photon absorption (red crossed squares) in the range 700-750 nm of amyloid fibrils and onephoton spectrum (dashed grey) re-plotted with the wavelength multiplied by a factor of three and normalized to the same peak height for comparison. The non-linear absorption data points for processes between $2 \mathrm{PA}$ and $3 \mathrm{PA}$ and $3<\mathrm{n}<5$ in range 625 and $930 \mathrm{~nm}$ (cf Figure 2) are plotted as the "effective 2PA" and denoted by blue triangles. 
References:

1 D. J. Selkoe. Folding proteins in fatal ways. Nature 426, 900-904 (2003).

2 F. Chiti \& C. M. Dobson. Protein misfolding, functional amyloid, and human disease. Annu. Rev. Biochem. 75, 333-366 (2006).

3 R. M. Murphy. Peptide Aggregation in Neurodegenerative disease. Annu. Rev. Biochem. 4, 155-174 (2002).

4 J. C. Rochet \& P. T. Lansbury Jr. Amyloid fibrillogenesis: themes and variations. Cur. Op. in Struct. Biol. 10, 60-68 (2000).

5 M. Sheikh-bahae, A. A. Said, T. Wei, D. J. Hagan \& E. W. van Stryland. Sensitive Measurement of Optical Nonlinearites Using a Single Beam. IEEE J. Quantum Electr. 26, 760-769 (1990).

6 E. Collini. Cooperative effects to enhance two-photon absorption efficiency: intra- versus inter-molecular approach. Phys. Chem. Chem. Phys. 14, 3725-3736 (2012).

7 S. B. Prusiner. Prions. PNAS 95, 13363-13383 (1998).

8 J. L. Jimenez, E. J. Nettleton, M. Bouchard, C. V. Robison \& C. M. Dobson. The protofilament structure of insulin amyloid fibrils. PNAS 99, 9196-9201 (2002).

9 J. F. Smith, T. P. J. Knowles, C. M. Dobson, C. E. MacPhee \& Welland, M. E. Characterization of the nanoscale properties of individual amyloid fibrils. PNAS 103, 15806-15811 (2006).

10 M. Samoc, J. P. Morrall, G. T. Dalton, M. P. Cifuentes \& M. G. Humphrey. Two-Photon and Three-Photon Absorption in an Organometallic Dendrimer. Angew. Chem. Int. Ed. 46, 731733 (2007).

11 T. Schwich, M. P. Cifuentes, P. A. Gugger, M. Samoc \& M. G. Humphrey. Electronic, Molecular Weight, Molecular Volume, and Financial Cost-Scaling and Comparison of Two-Photon Absorption Efficiency in Disparate Molecules. Adv. Mat. 23, 1433-1435 (2011).

12 M. Samoc et al. Two-photon absorption spectra and dispersion of the complex cubic hyperpolarizability $\gamma$ in organic and organometallic chromophores. in Multiphoton Processes in Organics and Their Application, I. Rau and F. Kajzar, eds, Old City Publishing Chapter 7, 341-355 (2011).

13 P. Hanczyc, B. Norden \& M. Samoc. Two-photon absorption of metal-organic DNA-probes. Dal. Trans. 41, 3123-3125 (2012).

14 Y. Meshalkin. Two-photon absorption cross sections of aromatic amino acids and proteins. Quan. Elec. 26, 536-537 (1996).

15 M. Samoc, A. Samoc \& J. G. Grote. Complex nonlinear refractive index of DNA. Chem. Phys. Lett. 431, 132-134 (2006).

16 D. Rativa, S. J. S. da Silva, J. Del Nero, A. S. L. Gomes \& R. E. de Araujo. Nonlinear optical properties of aromatic amino acids in the femtosecond regime. J. Opt. Soc. Am. B 27, 26652668 (2010).

17 R. L. Roberts et al. Organometallic Complexes for Nonlinear Optics. 45. Dispersion of the Third-Order Nonlinear Optical Properties of Triphenylamine-Cored Alkynylruthenium Dendrimers. Adv. Mat. 21, 2318-2322 (2009).

18 A. M. McDonagh, M. G. Humphrey, M. Samoc \& B. Luther-Davies. Organometallic Complexes for Nonlinear Optics. 17.1 Synthesis, Third-Order Optical Nonlinearities, and Two-Photon Absorption Cross Section of an Alkynylruthenium Dendrimer. Organomet. 18, 5195-5197 (1999).

19 S. J. Chung et al. Cooperative Enhancement of Two-Photon Absorption in Multi-branched Structures. J. Phys. Chem. B 103, 10741-10745 (1999).

20 M. Drobizhev et al. Strong Cooperative Enhancement of Two-Photon Absorption in DoubleStrand Conjugated Porphyrin Ladder Arrays. J. Am. Chem. Soc. 128, 12432-12433 (2006).

21 R. Friedman. Aggregation of amyloids in a cellular context: modelling and experiment. Biochem. J. 438, 415-426 (2011).

22 M. Reches \& E. Gazit. Casting Metal Nanowires Within Discrete Self-Assembled Peptide Nanotubes. Scien. 300, 625-627 (2003). 
23 E. P. Lundberg et al. Nanofabrication yields. Hybridization and click-fixation of polycyclic DNA nanoassemblies. ACS Nano 5, 7565-7575 (2011).

24 M. Fändrich, M. A. Fletcher \& C. M. Dobson. Amyloid fibrils from muscle myoglobin. Nature 410, 165-166 (2001).

25 E. S. Voropai et al. Spectral properties of Thioflavin T and its complexes with amyloid fibrils. . J. Appl. Spectrosc. 70, 868-874. (2003).

26 J. H. Viles. Metal ions and amyloid fiber formation in neurodegenerative diseases. Copper, zinc and iron in Alzheimer's, Parkinson's and prion diseases. Coord. Chem. Rev. 256, 22712284 (2012).

27 W. D. McCubbin, C. M. Kay, S. Narindrasorasak \& R. Kisilevsky. Circular-dichroism studies on two murine serum amyloid A proteins. Biochem. J. 256, 775-783 (1988).

28 P. P. Markowicz et al. Modified Z-scan techniques for investigations of nonlinear chiroptical effects. Opt. Exp. 12, 5209-5014 (2004).

29 X. Wang et al. Noninvasive laser-induced photoacoustic tomography for structural and functional in vivo imaging of the brain. Nat. Biotech. 21, 803 - 806 (2003).

30 R. M. Williams, W. R. Zipfel \& W. W. Webb. Interpreting Second-Harmonic Generation Images of Collagen I Fibrils. Biophys. J. 88, 1377-1386 (2005).

Acknowledgment: This work was sponsored under a Foundation for Polish Science "Welcome" grant to M.S. and a European Research Council (ERC) advanced senior grant to B.N.

Author contributions: P.H. conceived and carried out the experiments. P.H. M.S. and B.N. analyzed the results and authored the manuscript.

Additional information: Supplementary information is available in the online version of the paper.

Correspondence should be addressed to B.N. 


\title{
Supporting Information
}

\section{Multi-photon Absorption in Amyloid Protein Fibres}

\author{
Piotr Hanczyc ${ }^{\mathrm{a}, \mathrm{b}}$, Marek Samoc ${ }^{\mathrm{b}}$, Bengt Norden ${ }^{\mathrm{a}}$ * \\ ${ }^{\mathrm{a}}$ Department of Chemical and Biological Engineering, Chalmers University of Technology, \\ SE-41296 Gothenburg, Sweden \\ ${ }^{b}$ Institute of Physical and Theoretical Chemistry, Wroclaw University of Technology, 50-370, \\ Wroclaw, Poland \\ *corresponding author: norden@chalmers.se
}

\section{Two-photon absorption - imaginary part of $2^{\text {nd }}$ hyperpolarizability.}

Two-photon absorption (2PA), predicted theoretically 80 years ago by Göppert-Mayer ${ }^{1}$, was experimentally observed much later ${ }^{2}$ with the advent of lasers able of creating conditions with enough radiation intensity for achieving noticeable 2PA. Despite that basic principles were understood, successful attempts to theoretically deal with bigger systems than atoms or small molecules have been made first recently. Still, problems remain, depending on the approximations made and methods applied, as to conclusions what variations at a molecular level are the origin of enhanced 2PA observed in more complex systems of molecules such as those reported here.

For one-photon absorption (OPA) first-order perturbation theory is used to derive the equation for rate of change. Two common approximations are: 1) neglect of quadratic term of vector potential (A:A) and 2) the dipole approximation. The latter may be relaxed, for example when considering circular dichroism for electric dipole-forbidden, magnetic dipole-allowed transitions, by introducing magneticdipole and quadrupole transitions. The various cases of two-photon absorption and emission are most easily dealt with using Feynman diagrams ${ }^{3}$ where the terms in A:A, in first order, for the two photons cases are: 


$$
\frac{<m|A: A| n>}{\left[\omega_{m n}+\left(\omega_{2}+\omega_{1}\right)\right]}
$$

and

$$
\frac{<m|A: A| n>}{\left[\omega_{m n}+\left(\omega_{1}-\omega_{2}\right)\right]}
$$

Here Eq (2) corresponds to two-photon absorption. By contrast, the A:A term in the interaction is ineffective in two-photon absorption in the case of Eq (1) which corresponds to elastic (Rayleigh) scattering ${ }^{3}$.

Macroscopically two-photon absorption is related to the imaginary part of the "third-order susceptibility", i.e. the coefficient in the power expansion of the electric field dependent polarization

$$
P=\varepsilon_{0}\left(\chi^{(1)} E+\chi^{(2)} E^{2}+\chi^{(3)} E^{3} \quad \ldots\right)
$$

On the molecular level $\chi^{(1)}$ corresponds to the polarizability $\alpha$ and $\chi^{(2)}$ to the hyperpolarizability $\beta$ and $\chi^{(3)}$ to the "second hyperpolarizability" $\gamma$ which is developed in Luo et al. 2000. ${ }^{4}$ Summation over states provides:

$$
\begin{gathered}
\gamma\left(-\omega_{0}, \omega_{1}, \omega_{2}, \omega_{3}\right)=h^{-3} \sum P_{-0,1,2,3} \times \\
\sum_{k l m} \frac{<0|\mu| k><k|\mu| l><l|\mu| m><m|\mu| 0>}{\left(\omega_{k}-\omega_{0}+i \Gamma_{\mathrm{k}}\right)\left(\omega_{I}-\omega_{2}-\omega_{3}+i \Gamma_{1}\right)\left(\omega_{m}-\omega_{3}+i \Gamma_{\mathrm{m}}\right)} \\
-\sum_{k l} \frac{<0|\mu| k><k|\mu| 0><0|\mu| l><l|\mu| 0>}{\left(\omega_{k}-\omega_{0}+i \Gamma_{\mathrm{k}}\right)\left(\omega_{l}+\omega_{2}-\omega_{3}+i \Gamma_{1}\right)\left(\omega_{l}-\omega_{3}+i \Gamma_{\mathrm{m}}\right)}
\end{gathered}
$$

As shown by Perez-Moreno et al. ${ }^{5}$, a generalized Thomas-Kuhn sum rule may be used to derive a correspondence of $\mathrm{Eq}(4)$ without dipole approximation.

Before we elaborate on cooperative effects that could enhance the hyperpolarizability, let us note an interesting connection between the non-linear phenomena of two-photon absorption and the optical 
Kerr effect (OKE) i.e. the effect that strong photon fields induce anisotropy in polarizable media. OKE is a refractive correspondence to the non-linear absorptive effects defined by the second hyperpolarizability. The effect, sometimes called the "Buckingham effect", was first predicted by A.D. Buckingham ${ }^{6}$ in England in 1956 and experimentally pioneered by S. Kielich ${ }^{7}$ in Poland in 1959. Part of the OKE is due to (instantaneous) electron polarization along the electric field vector of the laser light, part is due to molecular orientation: the polarizable molecule gets upon interaction with the photon field a transient induced dipole moment leading to an orientational Boltzmann redistribution to minimize the energy $\boldsymbol{\mu} \cdot \mathbf{E}$ between the molecular induced dipole moment and the optical field. Just like the normal linear absorption coefficient is proportional to the imaginary part of the (complex) refractive index, there is a relation between the refractive nonlinear effect of $\chi^{(3)}$ and the nonlinear (two-photon) absorption: the latter is given by the imaginary part of the former. Thus, in molecular terms $2 \mathrm{PA}$ is the imaginary part of the second hyperpolarizability.

\section{Cooperative enhancement of 2PA.}

Without going into any detailed mechanisms it has been observed that molecules with high and anisotropic polarizability rapidly increase their dielectric permittivity when in concentrated compared to dilute form: carbon disulfide was early observed to increase its macroscopic permittivity (real as well as imaginary parts of the refractive index) presumably by a cooperative effect to self-polarization due to mutual interaction of transient induced dipoles, i.e. by dispersion forces ${ }^{8}$. Likewise we can expect that dimers, oligomers and aggregates of polarizable molecules will display cooperativity effects, both in their linear optical properties as well as in their non-linear properties.

Following the formalism above (Eq 4), applying second order perturbation theory and the dipole approximation, we have for the 2PA (second hyperpolarizability) a product of four transition moments. In the simplified case of three states only, the ground state 0 , some intermediate state $i$ and the final state $\mathrm{f}$, which is a one-photon-forbidden but two-photon-allowed symmetric $(\mathrm{g})$ state, the 2PA cross section has been derived as proportional to ${ }^{9,10}$

$$
\frac{|<i| \mu|0>|^{2}|<f| \mu|i>|^{2} \cos ^{2} \alpha}{\left(\frac{2 v_{i 0}}{v_{f 0}}-1\right)^{2} \Gamma_{f}}
$$


With $<m|\mu| n>$ the corresponding electric transition dipole moment between state $m$ and $n, v_{n m}$ the corresponding transition frequency, and $\Gamma_{\mathrm{f}}$ is the line width of state $\mathrm{f} . \alpha$ is the angle between the two transition moments in the expression.

Obviously, the mutual orientations of the transition moments by through-bond interactions in a molecule can tune the size of 2PA. As will be inferred below an analogous relationship to transition moments of separate chromophores could explain enhancement from through-space intermolecular interactions. Indeed, theoretical studies indicate that the cooperative effects due to such excitonic interactions are more efficient in enhancing two-photon absorption than are various extensions of the electronic (through bond) system of singular molecules ${ }^{11}$.

Consider two chromophores that are arranged in a dimeric structure side by side, for example, two benzoic acid molecules.

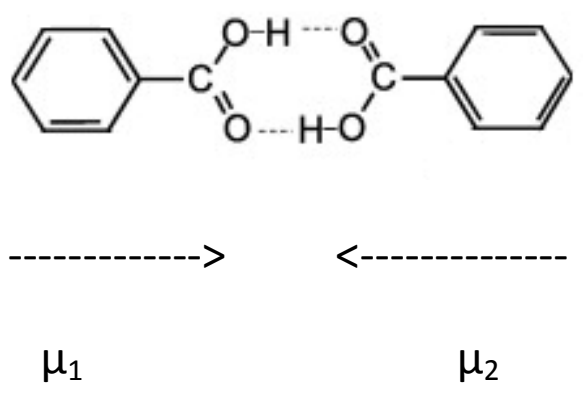

Fig. S1 Dimeric structure of benzoic acid. Symmetric exciton combination, forbidden in OPA but allowed in 2PA.

The first excited state $\mid i>$ has charge-transfer character and a transition moment $<i|\mu| 0\rangle$ directed from the nucleus of the benzene ring towards the carbonyl bond. The (through-space) dipolar exciton interactions give rise to two new excited states separated in energy by

$$
V=\frac{2|<i| \mu|0>|^{2}}{R^{3}}
$$

The low-energy state is accessible by OPA and with a transition moment equal to the sum of the monomeric transition moments. It accounts for a substantial red-shift of the normal absorption 
spectrum of benzoic acid upon dimer formation. The transition to the high-energy state should be OPA forbidden but 2PA allowed, providing an example of how side by side structures like that above could give rise to combined excitonic transitions with a parity promoting two-photon excitation (to our best knowledge benzoic acid dimer has not yet been studied with respect to 2PA). The excitonic model can be extended to large aggregates as have been reviewed by Collini and others ${ }^{11}$.

For the case of the protein fibres any discussion of possible mechanism for enhancement of 2PA will necessarily be very speculative since detailed information about their structures is generally missing. However, we have some indications about the average orientations of tyrosines in some of those fibres obtained by flow linear dichroism (LD) spectroscopy experiments ${ }^{12}$ which indicate that the benzene ring has a preferred orientation with its plane parallel with the fibre dimensions (positive LD sign of $\mathrm{L}_{b}$ transition) while the supporting bond to the amino acid backbone is at a more perpendicular orientation to the fibre axis (negative $\mathrm{LD}$ of $\mathrm{L}_{\mathrm{a}}$ band). Interestingly, at least qualitatively quite similar orientations have been recently also found for a series of prion yeast mutant (A. Reymer, K. Frederick, S. Lindquist and B. Nordén, to be published) indicating average orientations of the $\mathrm{L}_{b}(277 \mathrm{~nm})$ and $\mathrm{L}_{\mathrm{a}}$ $(230 \mathrm{~nm})$ transition dipole moments at about $40-50^{\circ}$ and $60-70^{\circ}$ to the fibre axis, respectively. Our 2PA results suggest that it is the first transition $\left(\mathrm{L}_{\mathrm{b}}=\mathrm{A}_{1 \mathrm{~g}} \rightarrow \mathrm{B}_{2 \mathrm{u}}\right)$ of tyrosine that is the one being strongly enhanced. This observation is in agreement both with the fact that $\mathrm{L}_{\mathrm{b}}$ being symmetry forbidden in benzene for normal absorption, although vibronically allowed by an $\mathrm{e}_{2 \mathrm{~g}} 606 \mathrm{~cm}^{-1}$ vibration, is allowed for 2PA: coparallel or near coparallel transition moments will increase the effect (see Eq (5) ) both in through-bond as well as through-space (exciton) mechanisms. In the benzenoid chromophore the next transition with a transition moment parallel with the vibronically allowed $L_{b}$ $\left(\mathrm{B}_{2 \mathrm{u}}\right)$ one, is the $\mathrm{B}_{1 \mathrm{u}}$ transition at $200 \mathrm{~nm}$, which is also vibronically allowed by vibrations $\left(1595 \mathrm{~cm}^{-1}\right)$ coupling it with a symmetry-allowed transition at $180 \mathrm{~nm}$. While there is experimental evidence for through-bond interactions leading to cooperative enhancement of $2 \mathrm{PA}$ in various conjugated aggregates of aromatic compounds $s^{10,11,13}$, and theoretical support too ${ }^{14}$, the importance of internal cooperative 2PA enhancement within the tyrosine (or other aromatic chromophores) seems less probable for various reasons, one being the large energy separation between the mixing states, another being the fact that the effect is so much weaker in the isolated chromophore system.

We also consider through-bond cooperative effects between different chromophores as less efficient, since the distances between adjacent aromatic chromophores through the peptide chain is too long and, furthermore, the conjugation broken in many places, including at the connections of the aromatic side groups. Experimental studies of conjugated porphyrin dimers suggest that both extended conjugation and resonance three-level conditions are contributing to $2 \mathrm{PA}$ enhancement ${ }^{15,16}$. 
Against this background we propose the exciton mechanism, schematically suggested above, as the remaining most plausible explanation of $2 \mathrm{PA}$ enhancement in protein fibrils. In view of the preferential alignment to $\mathrm{L}_{\mathrm{b}}$ transition moments parallel with the fibrous axis, we tentatively propose a cooperative effect operating via exciton combinations of $\mathrm{L}_{b}$ transition moments as sketched in Scheme 1 in the text. Many different exciton coupling modes involving different number of chromophores may contribute. The figure just represents one case of symmetric (in-phase) combinations which we believe could be efficient.

\section{Fibrils preparation for Z-scan measurements}

Insulin from bovine pancreas and lysozyme from chicken egg white was purchased from Sigma Aldrich dissolved in $\mathrm{pH}=2(0.01 \mathrm{M} \mathrm{HCl})$ water buffer and used without further purification. $\alpha$ synuclein was purchased from rPeptide and dissolved in 1xPBS buffer. Fibrils were prepared according to the protocols described elsewhere ${ }^{17,18}$. Samples concentration for nonlinear experiments were adjusted to $5 \mathrm{mg} / \mathrm{ml}$ if not otherwise stated. Nonlinear absorption experiments were carried out using a laser system consisting of a Quantronix Integra-C regenerative amplifier operating as an 800 nm pump and a Quantronix-Palitra-FS BIBO crystal-based optical parametric amplifier. This system delivers wavelength tunable pulses of $\sim 130$ fs length and was operated at the repetition rate of $1 \mathrm{kHz}$. Samples were placed in $1 \mathrm{~mm}$ path length Starna quartz glass cuvettes, stoppered, and sealed with Teflon tape. Results obtained on the cells with insulin (from bovine pancreas), lysozyme (from chicken egg white) and $\alpha$-synuclein solutions were calibrated against $Z$-scan measurements performed on a fused silica plate ( $4.66 \mathrm{~mm}$ thick) and compared with the measurements on an identical glass cell filled with the solvents alone: $\mathrm{pH}=2$ water buffer for insulin and lysozyme and $10 \mathrm{mM}$ PBS for $\alpha$ synuclein respectively. The output from the Palitra in the range from $525 \mathrm{~nm}$ to $950 \mathrm{~nm}$ was appropriately filtered using wavelength separators and colour glass filters to remove unwanted wavelength components, attenuated to $\mu \mathrm{J} /$ pulse range and used as excitation source for simultaneous recording of standard open-aperture $(\mathrm{OA})$ and closed-aperture $(\mathrm{CA}) \mathrm{Z}$-scan traces. The beam was focused so as to provide a focal spot in the range $\mathrm{w}_{0} \approx 25-50 \mu \mathrm{m}$ (giving the Rayleigh range which was always taken well in excess of the total thickness of the cell or the reference silica plate) and the cuvette was made to travel in the Z- direction, typically from -15 to $15 \mathrm{~mm}$. The data were collected using three InGaAs photodiodes (Thor Labs Inc.) that monitored the laser input, the OA signal and the CA signal, respectively. The outputs were fed into three channels of a digital oscilloscope and the data were collected by a computer using custom LabVIEW software. The traces of the CA and OA scans obtained by dividing each of them by the laser input reference were analyzed with the help of a custom fitting program that used equations derived by Sheik-Bahae et al. ${ }^{19}$ (Figs S2 and S3 show examples of fitted closed aperture and open aperture curves for the case of predominantly 2PA and 3PA character of the absorption). The NLO properties of the fibrils were computed assuming additivity of the 
nonlinear contributions of the solvent and the solute and the applicability of the Lorentz local field approximation $^{20}$. The nonlinear absorption was calculated as values of the two-photon cross section $\sigma_{2}$ and, where the shapes of the Z-scans indicated predominance of the three-photon absorption process, as values of the three-photon cross sections $\sigma_{3}$. The two-photon absorption cross sections were scaled using the molecular weight $\mathrm{M}$, into $\sigma_{2} / \mathrm{M}$ values as discussed recently ${ }^{21}$.

The open aperture curves in the wavelength ranges where neither two-photon nor three-photon behaviour provided a good fit were treated in the following manner. First, an "effective" value of the two-photon absorption cross section was determined by adjusting the theoretical curve in such a way that the depth of the dip corresponded to the experimental curve and neglecting the inadequacy of the width of the dip. Subsequently, the width of the dip was adjusted by treating the exponent $n$ in the nonlinear Beer's law $\mathrm{d} I / \mathrm{d} z=-k I^{n}$ as a fitting parameter. The values of $n$ required to reach a good fit are presented in Fig. 2. The "effective" values of $\sigma_{2}$ obtained in the previous step were then adjusted to their expected values at the intensity of $100 \mathrm{GW} / \mathrm{cm}^{2}$. The required values of the actual peak light intensities in the Z-scan experiment were computed from the closed-aperture Z-scan results obtained for the fused silica plate.

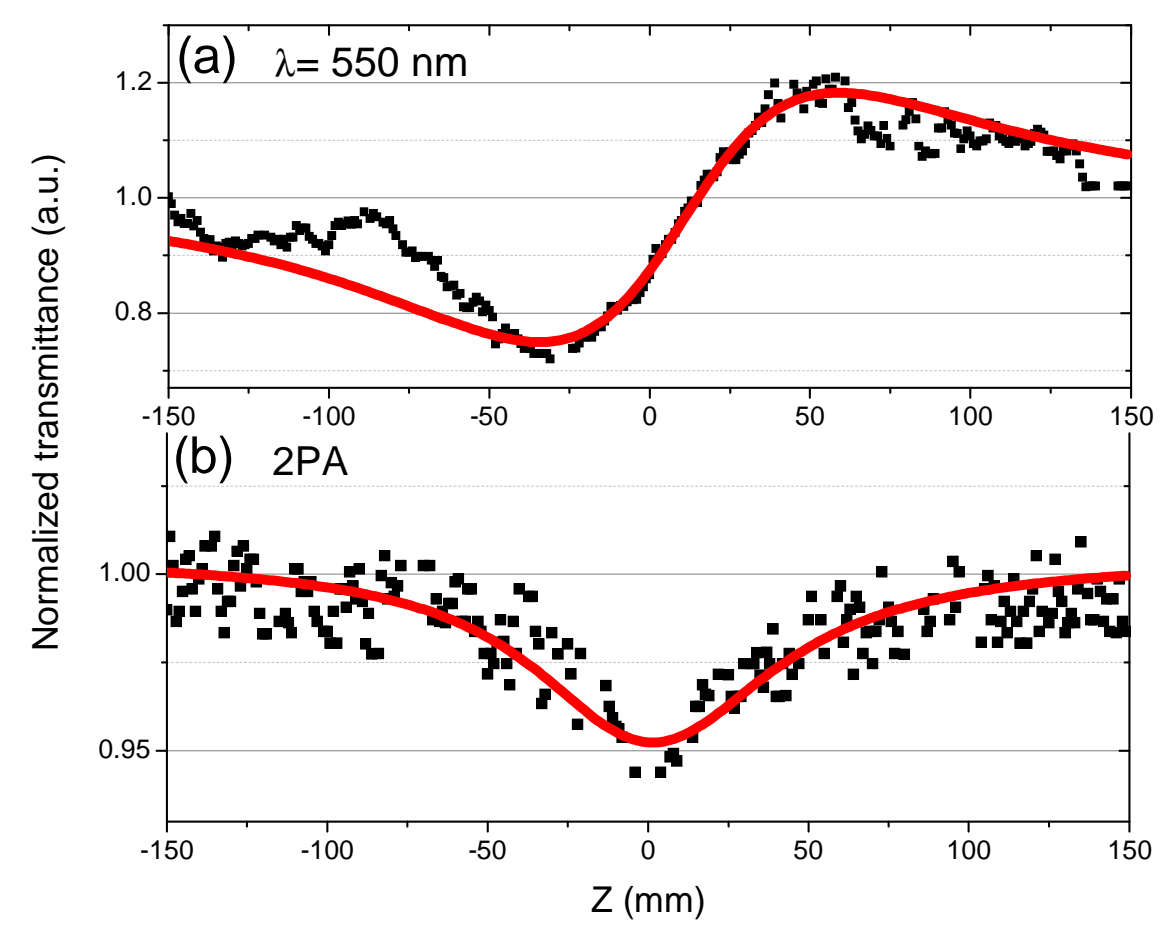

Fig. S2 Closed (a) and open (b) aperture Z-scan at $550 \mathrm{~nm}$ of amyloid fibrils (black squares) and theoretical 2PA (red solid line) fit. 


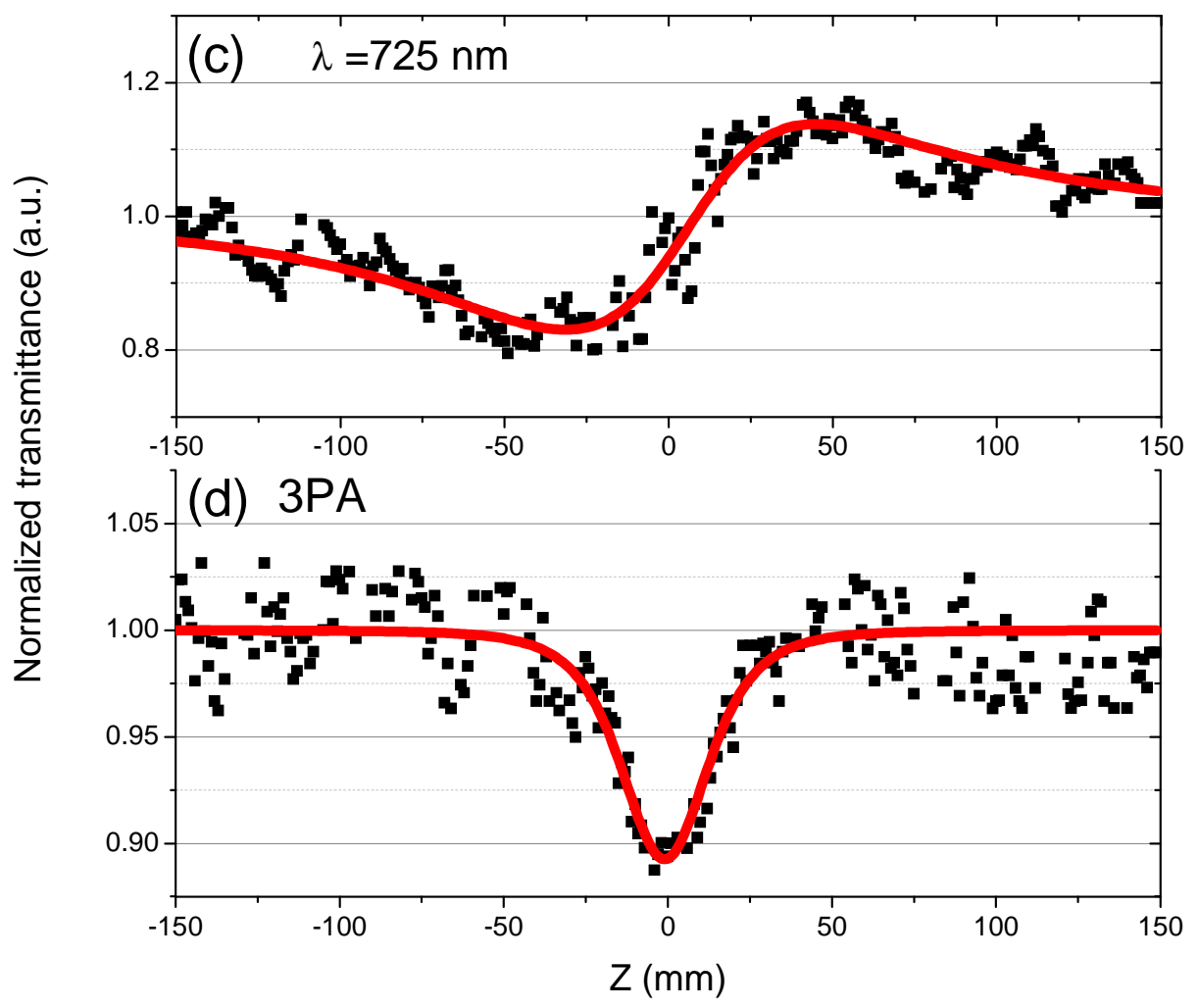

Fig. S3 Closed (c) and open (d) aperture Z-scan at $725 \mathrm{~nm}$ of amyloid fibrils (black squares) and theoretical 3PA (red solid line) fit. 

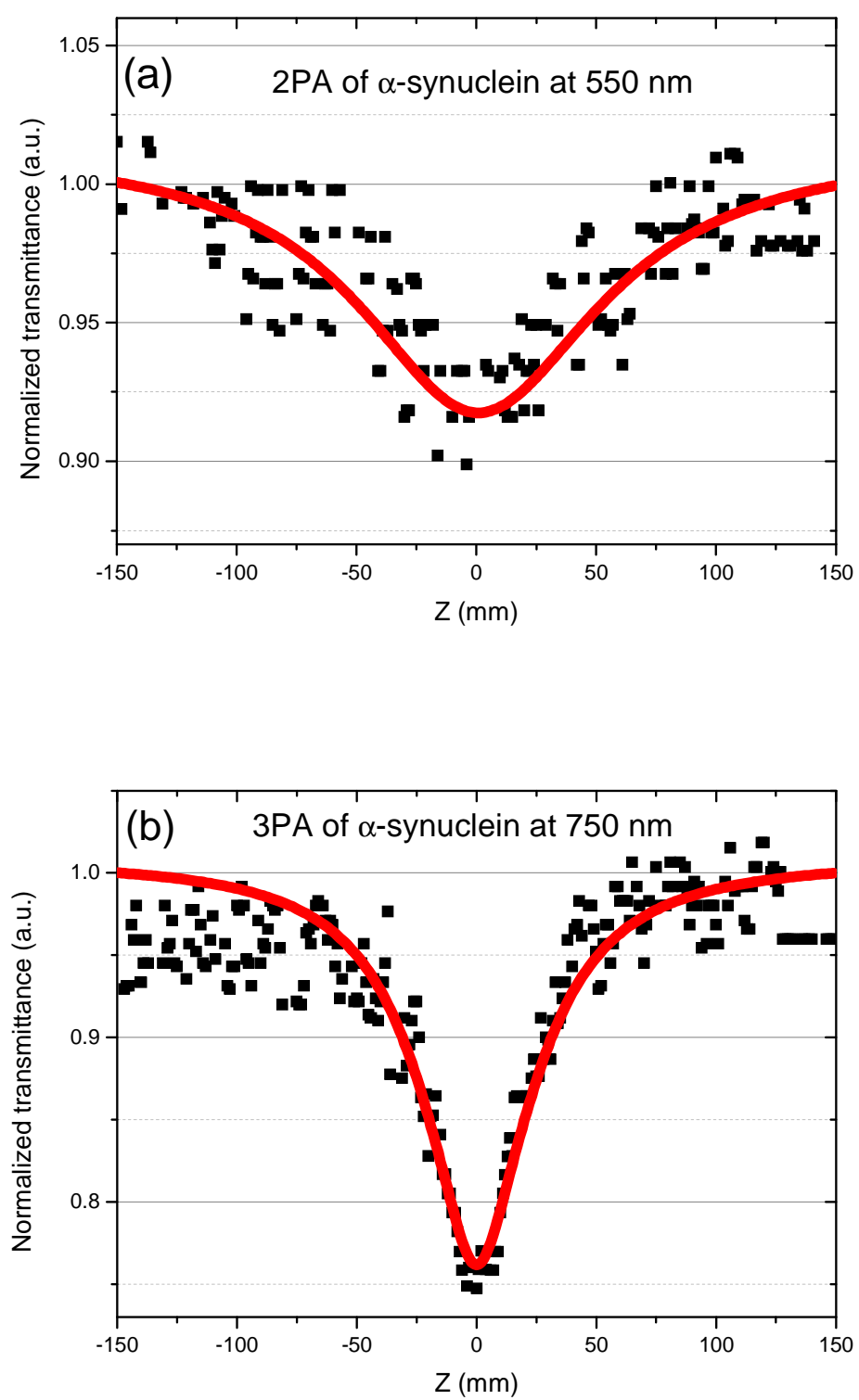

Fig. S4 Open aperture Z-scan at $550 \mathrm{~nm}$ in 2PA region (a) and $750 \mathrm{~nm}$ in 3PA region (b) of amyloid fibrils from $\alpha$-synuclein protein (black squares) and theoretical fit (red solid line). 


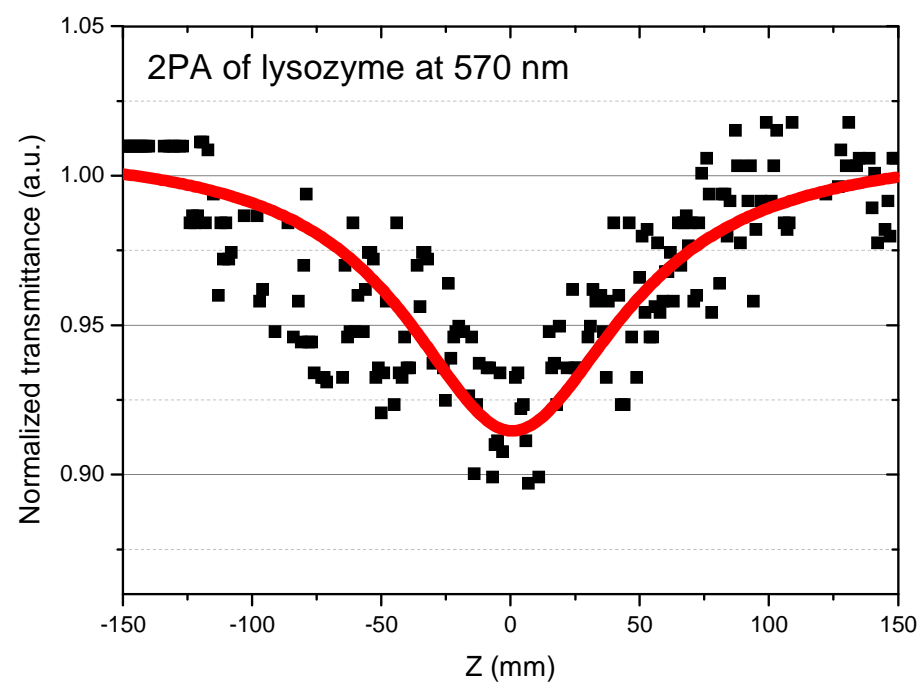

Fig. S5 Open aperture Z-scan at $570 \mathrm{~nm}$ of amyloid fibrils from lysozyme protein (black squares) and theoretical fit (red solid line). 


\section{Pump-probe experiment}

The Z-scan experiment is essentially lacking the temporal resolution and thus not allowing to distinguish between instantaneous multiple photon absorption processes and those involving slower effects. Therefore, a preliminary time-resolved pump-probe experiment has been performed to prove the instantaneous character of the absorption. The experiment was carried out using both the pump and the probe at $800 \mathrm{~nm}$. The beam originating from the Integra regenerative amplifier was attenuated and split into two parts, one of them providing the pump the other being the probe. The probe beam path involved a computer controlled delay line and the pump beam was modulated by a mechanical chopper. Both beams were focused with a single $50 \mathrm{~mm}$ diameter lens onto a $1 \mathrm{~mm}$ cell containing the sample. The transmitted probe modulation was then detected by a photodiode and a lock-in amplifier. Fig. S6 shows the time-resolved pump-probe signal. It was verified that this signal was much stronger than any signals obtainable from the cuvette with the solvent alone. The shape of the signal was fitted assuming that the laser pulse is of $\operatorname{sech}^{2}$ type with the FWHM of $159.6 \mathrm{fs}$. Only a slight asymmetry of the signal was detected which was accounted for by convoluting in a small admixture of a delayed component (modeled as a multiphoton excited state absorption process) with the relaxation time of 797 fs. Since the signal is mostly composed of the instantaneous components, it can be attributed to multiphoton absorption occurring on the temporal overlap of the pump and probe beams.

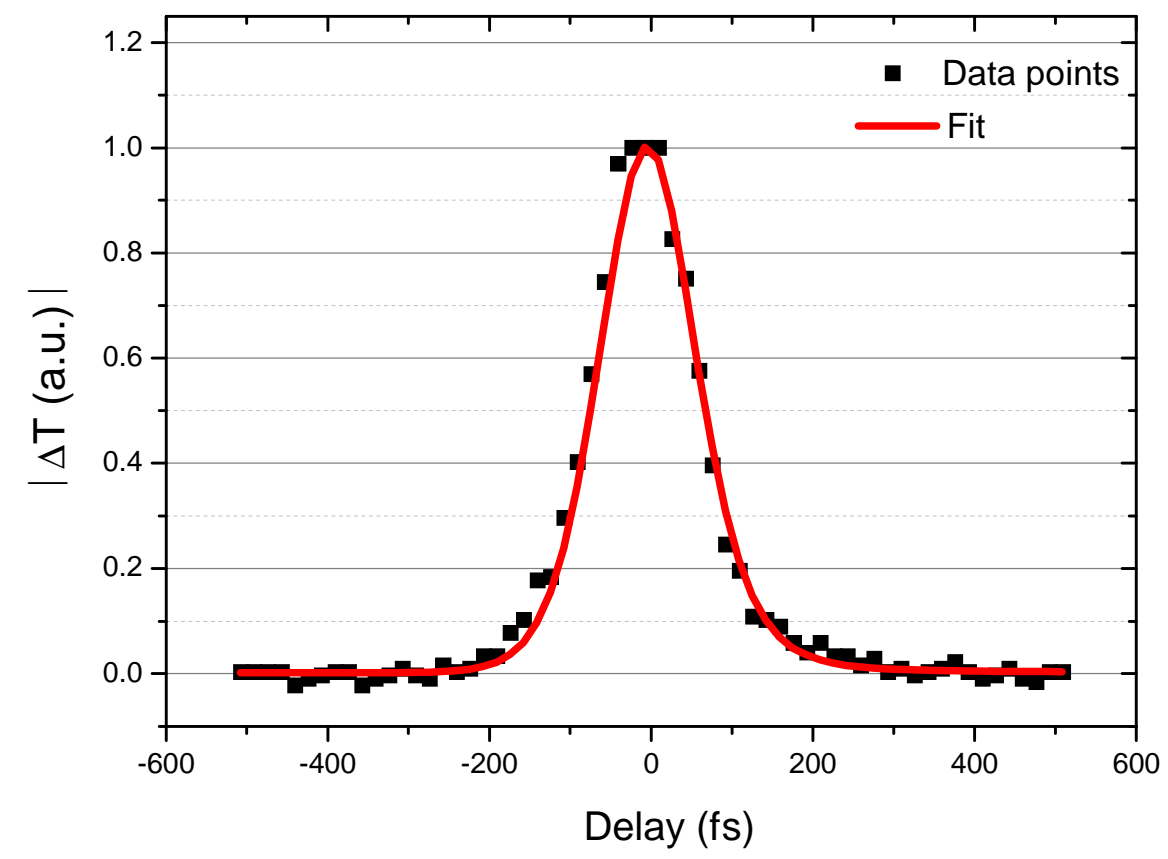

Fig. S6 Example of pump-probe experiment on insulin fibrils at $800 \mathrm{~nm}$ 
Sequence composition

Insulin

A-Chain:

GIVEQCCASVCSLYQLENYCN

B-Chain:

FVNQHLCGSHLVEALYLVCGERGFFYTPKA

$\alpha$-synuclein:

\begin{abstract}
MDVFMKGLSK AKEGVVAAAE KTKQGVAEAA GKTKEGVLYV GSKTKEGVVH GVATVAEKTK EQVTNVGGAV VTGVTAVAQK TVEGAGSIAA ATGFVKKDQL GKNEEGAPQE GILEDMPVDP DNEAYEMPSE EGYQDYEPEA
\end{abstract}

Lysozyme

KVFGRCELAAAMKRHGLDDYRGYSLGDWVCAAKFESDFDTDRDTDGSTDYGILEID SRWWCDDGRTPGSRDLCDIPCSALLSSDITASVDCAKKIVSDGDGMDAWVAWRDRC KGTDVEAWIRGCRL

1 M. Goeppert-Mayer. Über Elementarakte mit zwei Quantensprüngen. Ann. Phys. 9, 273-295 (1931).

2 W. Kaiser \& C. G. B. Garrett. Two-Photon Excitation in $\mathrm{CaF}_{2}: \mathrm{Eu}^{2+}$. Phys. Rev. Lett. 7, 229-231 (1961).

3 J. I. Steinfeld. Molecules and radiation : an introduction to modern molecular spectroscopy. 2nd edition 4th printing M.I.T Press Boston 1993 Figure 13.8

4 Y. Luo, P. Norman, P. Macak \& H. Agren. Solvent-Induced Two-Photon Absorption of a PushPull Molecule. J. Phys. Chem. A 104, 4718-4722 (2000).

5 J. Pérez-Moreno, K. Clays \& Kuzyk, M. G. A new dipole-free sum-over-states expression for the second hyperpolarizability. J. Chem. Phys. 128, 084109 (2008).

6 A.D. Buckingham. Birefringence Resulting from the Application of an Intense Beam of Light to an Isotropic Medium Proc. Phys. Soc. Sec. B 69, 344-349 (1956).

7 S. Kielich \& A. Piekara. A Statistical Molecular Theory of Electric, Magnetic and Optical Saturation Phenomena in Isotropic Dielectric and Dia-Magnetic Media. Acta Phys. Polon. 18, 439-471 (1959).

8 A. Davidsson \& B. Nordén. A Method for Determination of the Refractive Index in a Region of Absorption. Anomalous Dispersion of CS2 in the UV Range. Chem. Phys. 8, 223-230 (1975).

9 M. Drobizhev et al. Understanding Strong Two-Photon Absorption in $\pi$-Conjugated Porphyrin Dimers via Double-Resonance Enhancement in a Three-Level Model. J. Am. Chem. Soc. 126, 15352-15353 (2004).

10 M. Drobizhev et al. Strong Cooperative Enhancement of Two-Photon Absorption in DoubleStrand Conjugated Porphyrin Ladder Arrays. J. Am. Chem. Soc. 128, 12432-12433 (2006).

11 E. Collini. Cooperative effects to enhance two-photon absorption efficiency: intra- versus inter-molecular approach. Phys. Chem. Chem. Phys. 14, 3725-3736 (2012). 
B. Nordén, A. Rodger \& T. Dafforn. Linear Dichroism and Circular Dichroism. A Textbook on Polarized-Light Spectroscopy. ISBN: 978-1-84755-902-9 British Library, RSC (2010).

$13 \mathrm{H}$. Y. Woo et al. Solvent Effects on the Two-Photon Absorption of Distyrylbenzene Chromophores. J. Am. Chem. Soc. 127, 14721-14729 (2005).

14 P. Macak, Y. Luo \& H. Ågren. Simulations of vibronic profiles in two-photon absorption. Chem. Phys. Lett. 330, 447-456 (2000).

15 M. Drobizhev et al. Extremely strong near-IR two-photon absorption in conjugated porphyrin dimers: quantitative description with three essential states model. J. Phys. Chem. B 109, 7223-7236 (2005).

16 S. J. Chung et al. Cooperative Enhancement of Two-Photon Absorption in Multi-branched Structures. J. Phys. Chem. B 103, 10741-10745 (1999).

17 C. Kitts, T. Beke-Somfai \& B. Norden. Michler's Hydrol Blue: A Sensitive Probe for Amyloid Fibril Detection. Biochem. 50, 3451-3461 (2011).

18 K. A. Conway, J. D. Harper \& P. T. Lansbury. Fibrils Formed in Vitro from R-Synuclein and Two Mutant Forms Linked to Parkinson's Disease are Typical Amyloid. Biochem. 39, 2552-2563 (2000).

19 M. Sheikh-bahae, A. A. Said, T. Wei, D. J. Hagan \& E. W. van Stryland. Sensitive Measurement of Optical Nonlinearites Using a Single Beam. IEEE J. Quantum Electr. 26, 760-769 (1990).

20 M. Samoc et al. Two-photon absorption spectra and dispersion of the complex cubic hyperpolarizability $\gamma$ in organic and organometallic chromophores. in Multiphoton Processes in Organics and Their Application, I. Rau and F. Kajzar, eds, Old City Publishing Chapter 7, 341-355 (2011).

21 T. Schwich, M. P. Cifuentes, P. A. Gugger, M. Samoc \& M. G. Humphrey. Electronic, Molecular Weight, Molecular Volume, and Financial Cost-Scaling and Comparison of Two-Photon Absorption Efficiency in Disparate Molecules. Adv. Mat. 23, 1433-1435 (2011). 